\title{
Cardiovascular manifestations and echocardiographic findings in pediatric multisystem inflammatory syndrome associated with COVID-19 (MIS-C): A retrospective study
}

\author{
Jashvanth H J' Sudha Rudrappa ${ }^{2}$, Pratibha Manjunath Patagar ${ }^{3}$ \\ From ${ }^{1}$ Junior Resident, ${ }^{2}$ Professor and HOD, ${ }^{3}$ Senior Resident, Department of Paediatrics, Mysore Medical College and Research Institute, Mysore, \\ Karnataka, India
}

\begin{abstract}
Background: The multisystem inflammatory syndrome in children (MIS-C) has been described recently during COVID-19 pandemic. It is a delayed post-infectious response with a lag time of 4-6 weeks following the exposure to severe acute respiratory syndrome coronavirus-2 (SARS-CoV-2), involving multiple systems which is being increasingly reported worldwide. Aim: The study aims to investigate and report the cardiovascular manifestation and echocardiographic findings in pediatric patients diagnosed with MIS-C. Materials and Methods: The present study is a retrospective analysis of clinical data gathered from 40 admitted children diagnosed with MIS-C. The study was conducted for 1 year (from $1^{\text {st }}$ June 2020 to $30^{\text {th }}$ May 2021) at Cheluvamba Hospital, a tertiary care center attached to Mysore Medical College And Research Institute, Mysore, Karnataka. Results: Out of 40 children, 21 (52.5\%) had manifestation of cardiovascular system in the form of shock, requiring ionotropic support and care at the Intensive Care Unit. Among them, $90 \%$ patients show elevated cardiac biomarker (Troponin T), 33.3\% had two-dimensional echocardiographic (2D echo) findings in which $9.5 \%$ show coronary artery dilatation and $23.8 \%$ show left ventricular hypertrophy. Conclusion: MIS-C is a hyperinflammatory syndrome related to SARS-CoV-2 infection. Cardiac involvement is evidenced by perturbation of cardiac chamber size, function, coronary artery abnormalities, and elevated cardiac biomarker. Though, most of the patients responded to the initial treatment and recovered, there is scarcity of data regarding long-term cardiovascular complication.
\end{abstract}

Key words: 2D echocardiographic, COVID-19, Multisystem inflammatory syndrome in children, Troponin T

$\mathrm{T}$ The 2019 outbreak of corona virus infection in Wuhan has been attributed to severe acute respiratory syndrome coronavirus-2 (SARS-CoV-2) [1] and was termed as corona virus disease in 2019 (COVID-19) by the World Health Organization (WHO). It has spawned public health emergency of international concern [2]. SARS-CoV-2 infection in children is thought to be relatively mild compared to that in adults and most often remained asymptomatic or minimally symptomatic [3]. Since mid-April 2020, cluster of pediatric cases of severe systemic hyperinflammation epidemiologically linked to COVID-19 has been reported in increasing numbers. These increasing number of cases led to health advisory from Centers for Disease Control and Prevention and WHO which identified these cases as a novel condition named multisystem inflammatory syndrome in children (MIS-C) or pediatric multisystem inflammatory syndrome [4]. Till now, there is limited awareness regarding SARS-CoV-2 related

\section{Access this article online}

Received - 20 September 2021

Initial Review - 09 October 2021

Accepted - 06 November 2021

DOI: $10.32677 /$ ijch.v8i11.3130 cardiovascular injury in pediatric population. However, recently a large number of children hospitalized in Intensive Care Unit (ICU) for cardiogenic shock or acute left ventricular dysfunction in setting of MIS-C secondary to COVID-19.

\section{MATERIALS AND METHODS}

Children and adolescents aged between 0 and 18 years with diagnosis of MIS-C were included in the study. Patients, in whom an alternative diagnosis (including bacterial sepsis, staphylococcal or streptococcal shock, Kawasaki disease and tropical infection such as dengue, malaria, and rickettsial fever etc.) was made, were excluded from the study.

The study was approved by ethical committee of our institution. We retrospectively reviewed clinical data of 40 admitted children in Cheluvamba hospital, a tertiary care center attached to Mysore Medical College and Research Institute, Mysore. All subjects met the diagnostic criteria of MIS-C given by the WHO [5].

Correspondence to: Dr. Jashvanth H J, Department of Paediatrics, Mysore Medical College and Research Institute, $10787^{\text {th }}$, Cross $3^{\text {rd }}$ Stage Dattagahalli, Mysore - 570 022, Karnataka, India. E-mail: jashvanthjashu871@gmail.com

(C) 2021 Creative Commons Attribution-NonCommercial 4.0 International License (CC BY-NC-ND 4.0). 
The case definition includes children and adolescents with fever $>=3$ days AND any two of the following: (1) Rash or bilateral non purulent conjunctivitis or muco cutaneous inflammation (oral, hand or feet), (2) Hypotension or shock, (3) Features of myocardial dysfunction, pericarditis, valvulitis or coronary abnormalities including echo findings or elevated troponin or $\mathrm{N}$-terminal pro b-type natriuretic peptide, (4) Evidence of coagulopathy (deranged prothrombin time, partial thromboplastin time, elevated D-dimer), (5) Acute gastrointestinal problems such as diarrhea, vomiting, and pain abdomen AND elevated marker of inflammation such as erythrocyte sedimentation rate, C-reactive protein (C-RP), procalcitonin AND evidence of COVID-19 (Reverse transcription-polymerase chain reaction (RT-PCR) or antigen test or serology positive) OR likely contact with COVID-19 patients AND no other obvious microbial cause of inflammation including bacterial sepsis, staphylococcal or streptococcal shock syndrome. Demographic characteristics, clinical data, laboratory findings, result of cardiovascular examination including electrocardiogram (ECG) and echocardiogram (Echo), medical treatment and clinical outcome was retrieved from patient files.

\section{RESULTS}

A total of 40 children were included in the study (median age 9 years; 23 males and 17 females). All patients presented with persistent fever $>38^{\circ} \mathrm{C}$ in a days before admission. Other common signs and symptoms are mentioned in the Table 1.

Children with cardiovascular manifestations (Fig. 1) in the form of shock required inotropic support for 4 days. Among them, one was presented with symptoms of cardiac failure and none of them had comorbidities or known cardiac disease. Cardiac biomarkers such as Troponin $\mathrm{T}$ were elevated in $90 \%$ children. The biochemical markers of inflammation were also raised in majority of the patients as presented in the Table 2 .

All patients were positive for SARS-CoV2 antibodies either IgG or IgM though nasopharyngeal swab for COVID RT-PCR was negative. In majority of the patients, peak biochemical abnormality was on admission with no significant difference between these parameters on admission versus peak level during hospitalization.

An abnormal ECG was seen in six (28.5\%) children, that includes abnormal ST and T wave and prolonged PR interval in one patient (Fig. 2).

2D Echo was done for all hospitalized patients, among which seven $(33.3 \%)$ had Echo findings, two $(9.5 \%)$ had coronary artery dilatation. The most commonly affected artery was left main coronary artery, none of them had coronary aneurysm, five (23.8\%) revealed left ventricular hypertrophy, and one (4.7\%) had mild pericardial effusion (Fig. 3).

All patients were treated according to the guidelines given by the ministry of health and family welfare India [6]. All patients with cardiovascular manifestations received immuno-modulatory therapy with intravenous immunoglobin $2 \mathrm{~g} / \mathrm{kg}$ over $24-48 \mathrm{~h}$, intravenous steroid and ionotropic support. $80 \%$ patients require dobutamine, $50 \%$ require adrenaline and $20 \%$ require dopamine. For all patients, common tropical infection was ruled out and broad spectrum antibiotics were given.
The clinical course of most of the patients was favorable. Mean duration of ICU care was 6 days with most of the patients discharged home and advised follow-up. Only one patient who presented initially with symptoms of cardiac failure was

Table 1: Clinical features of MIS-C

\begin{tabular}{lcc}
\hline Clinical features & Number & Percentage \\
\hline Fever & 40 & 100 \\
Vomiting & 29 & 72.5 \\
Cardio vascular manifestation-shock & 21 & 52.5 \\
Pain abdomen & 18 & 45 \\
Loose stools & 10 & 25 \\
Bilateral non purulent conjunctivitis & 7 & 17.5 \\
Erythematous skin rashes & 3 & 7.5 \\
\hline
\end{tabular}

MIS-C: Multisystem inflammatory syndrome in children

Table 2: Laboratory profile of MIS-C

\begin{tabular}{lccc}
\hline Laboratory parameter & Number & Percentage & Cut-off values \\
\hline LDH & 38 & 95 & $450 \mu / 1$ \\
Serum ferritin & 36 & 90 & $500 \mathrm{ng} / \mathrm{ml}$ \\
C-RP & 32 & 80 & $30 \mathrm{mg} / 1$ \\
Anemia & 25 & 62.5 & $10 \mathrm{mg} \%$ \\
Thrombocytopenia & 23 & 57.5 & $1.5 \mathrm{lakh}$ \\
Neutrophilia & 11 & 27.5 & $7500 / \mathrm{mm}^{3}$ \\
Lymphopenia & 9 & 22.5 & $1500 / \mathrm{mm}^{3}$ \\
Leucopenia & 8 & 20 & $4000 / \mathrm{mm}_{3}$ \\
D-dimer & 7 & 17.5 & $500 \mathrm{ng} / \mathrm{ml}^{2}$ \\
\hline
\end{tabular}

MIS-C: Multisystem inflammatory syndrome in children, LDH: Lactate dehydrogenase, C-RP: Reactive protein

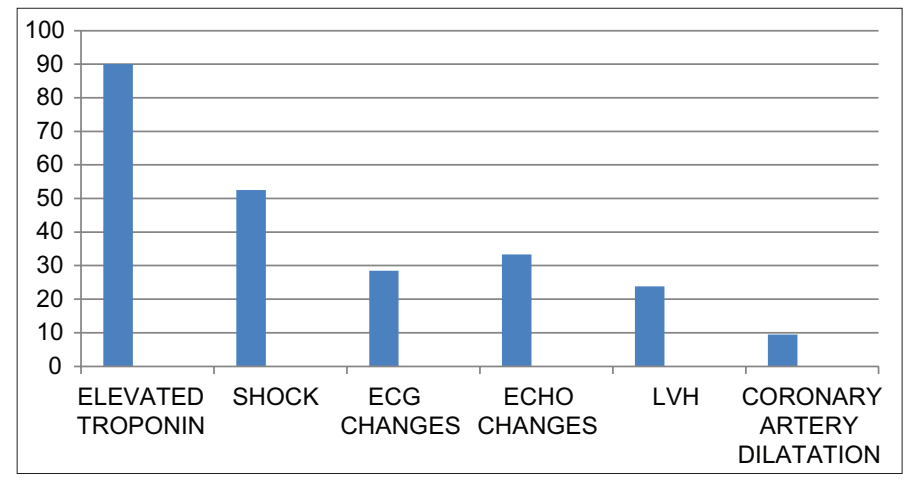

Figure 1: Cardiovascular manifestations

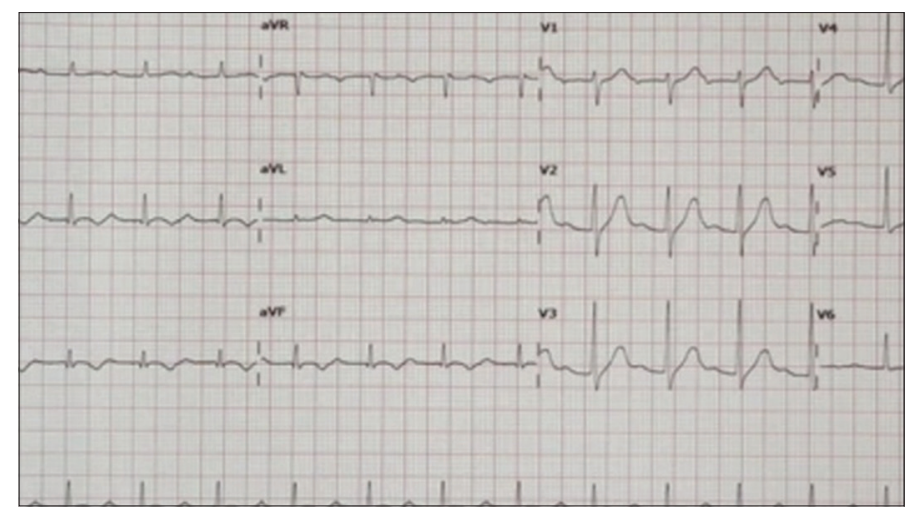

Figure 2: ECG showing abnormal PR interval and ST segment 


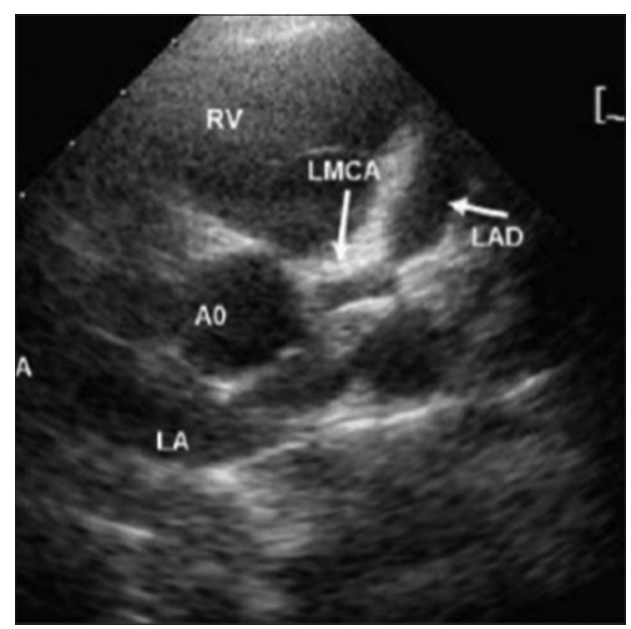

Figure 3: 2D echo showing dilated coronary artery

succumbed to death. All patients were followed up on out-patient basis; inflammatory markers and 2D Echo were repeated after 7-14 days post-discharge. None of them showed worsening of clinical condition or abnormal inflammatory markers.

\section{DISCUSSION}

MIS-C associated with COVID-19 is a hyper-inflammatory syndrome associated with COVID-19 infection. As the number of COVID cases increased in country, MIS-C cases also increased exponentially [7]. Children with MIS-C have been reported to have cardiac involvement and multiple mechanisms have been suggested for cardiac damage. It may be a part of systemic inflammatory syndrome with release of high level of cytokine that can injure multiple tissues including vascular endothelium and cardiac myocyte. Alternatively, SARS-CoV-2 virus may directly infect myocardium causing viral myocarditis or myocardial damage. The damage may also be caused by increased cardiac metabolic demand associated with systemic infection and hypoxia caused by severe pneumonia or acute respiratory distress syndrome [8].

Current study is a retrospective review of 40 children, of which around half the population showed cardiac involvement and all of them presented with shock requiring inotropic support and ICU care. Studies by Dhanalakshmi et al. [9], Jain et al. [7], and Israel et al. [10] had observed cardiovascular involvement in $63 \%, 65 \%$, and $68 \%$ patients, respectively, which is closely associated with our observation.

About $90 \%$ patients in current study had elevated inflammatory markers and cardiac biochemical marker, Troponin T. Similar result (93\%) was also observed in a study done by Israel et al. [10]. However, study done by Dhanalakshmi et al. [9], described a contradictory result where troponin $\mathrm{T}$ was elevated in only $16.6 \%$ of children.

We observed abnormal ECG in $28.5 \%$ children demonstrating abnormal ST segment, T wave and prolonged PR interval. Study by Israel et al. [10] show abnormal ST and $\mathrm{T}$ wave changes in $22 \%$ patients. Cardiac imaging, particularly $2 \mathrm{D}$ echo is of prime importance in evaluating cardiovascular system. In our study, 33.3\% patients showed 2D echo findings and $9.5 \%$ of the children showed coronary artery dilatation. Studies done by Dhanalakshmi et al. [9],
Shreepal Jain et al. [7] and Israel et al. [10], coronary artery dilatation was observed in $15.7 \%, 26 \%$ and $24 \%$ patients, respectively.

Clinical outcome of most of the patients was favorable. The sample size of MIS-C patients is an important limitation of this study, though our study has enrolled perhaps maximum number of patients for a tertiary care center collected over 12 months.

\section{CONCLUSION}

Follow-up of MIS-C patients is essential to better understand the evolution and prognosis of this disease. Further, studies are required to define evidence based management of this novel disease. Hence, a prospective study with larger sample size is essential to dissect the system-wise manifestations of the disease, and its immediate outcome and also the long term complications. Pediatricians should be aware of this new clinical syndrome related to SARS-CoV-2 infection. Early identification with employment of appropriate therapies is the key to favorable outcomes.

\section{REFERENCES}

1. Song W, Li J, Zou N, Guan W, Pan J, Xu W. Clinical features of pediatric patients with coronavirus disease (COVID-19). J Clin Virol 2020;127:104377.

2. World Health Organization-Home Health Topics Coronavirus; 20192020. Available from: http://www.who.int/health topics/coronavirus. [Last accessed on 2021 Nov 05].

3. Alsaied T, Tremoulet AH, Burns JC, Saidi A, Dionne A, Lang SM, et al. Review of cardiac involvement in multisystem inflammatory syndrome in children. Circulation 2021;143:78-88.

4. Sperotto F, Friedman KG, Son MB, VanderPluym CJ, Newburger JW, Dionne A. Cardiac manifestations in SARS-CoV-2-associated multisystem inflammatory syndrome in children: A comprehensive review and proposed clinical approach. Eur J Pediatr 2021;180:307-22.

5. World Health Organization-Multisystem Inflammatory Syndrome in Children and Adolescents Temporally Related to COVID-19, News Room>Detail-MISC Related to COVID-19; 2020. Available from: http:// www.who.int. [Last accessed on 2021 Nov 05].

6. Guidelines on Operationalization of COVID-19 in Children and AdolescentsManagement of COVID 19-Government of India: Ministry of Health and Family Welfare; 2021. p. 8. Available from: http://www.mohfw.gov.in. [Last accessed on 2021 Nov 05].

7. Jain S, Sen S, Lakshmivenkateshiah S, Bobhate P, Venkatesh S, Udani S, et al. Multisystem inflammatory syndrome in children With COVID-19 in Mumbai, India. Indian Pediatr 2020;57:1015-9.

8. Cardiac Manifestation of COVID-19, StatPearls-NCBI Book Shelf; 2019. Available from: http://www.ncbi.nim.gov. [Last accessed on 2021 Nov 04].

9. Dhanalakshmi K, Venkataraman A, Balasubramanian S, Madhusudan M, Amperayani S, Putilibai S, et al. Epidemiological and clinical profile of pediatric inflammatory multisystem syndrome-temporally associated with SARS-CoV-2 (PIMS-TS) in Indian children. Indian Pediatr 2020;57:1010-4.

10. Valverde I, Singh Y, Sanchez-de-Toledo J, Theocharis P, Chikermane A, Di Filippo S, et al. Acute cardiovascular manifestations in 286 children with multisystem inflammatory syndrome associated with COVID-19 infection in Europe. Circulation 2021;143:21-32.

Funding: None; Conflicts of Interest: None Stated.

How to cite this article: Jashvanth HJ, Rudrappa S, Patagar PM. Cardiovascular manifestations and echocardiographic findings in pediatric multisystem inflammatory syndrome associated with COVID-19 (MIS-C): A retrospective study. Indian J Child Health. 2021; 8(11):391-393. 